\title{
Fractal Spheres as Molecules for Artificial Dielectric Metamaterials
}

\author{
M. J. FACCHINE \\ D. H. WERNER
}

The Pennsylvania State University

Department of Electrical Engineering

University Park, Pennsylvania, USA

\begin{abstract}
The most common types of molecule geometries that have been considered in the past for artificial dielectric materials include simple dipoles and spheres. In this article we consider a fractal approach, combining aspects of both the dipole and sphere, resulting in an inclusion with a greater number of resonances as well as resonant behavior at lower frequencies than either their dipole or sphere counterparts of the same size. The results of this investigation suggest that the fractal sphere molecules allow an extension of the lower frequency band of artificial dielectric metamaterials and in some instances also provide multi-band response.
\end{abstract}

Keywords metamaterials, artificial dielectric, fractals, fractal trees, multi-band

\section{Introduction}

In recent years, the development of artificial dielectric metamaterials has led to advancements in a variety of areas including antennas, waveguides, polarizers, and electromagnetic absorbing materials. These artificial dielectric metamaterials are formed by introducing inclusions (i.e., molecules) into a background dielectric material. These inclusions influence the electromagnetic properties of the dielectric material of interest. It has been shown that the properties of these artificial dielectric metamaterials are strongest around the resonant frequencies of the inclusions (Mariotte, Tretyakov, \& Sauviac, 1996; Bahr \& Clausing, 1994). It is well known that the backscattering properties of simple dipoles and spheres are characteristically narrowband (Harrington, 1968, 1961). Different types of inclusions require different mixing rules depending on properties of the host dielectric and characteristics of the inclusions, such as shape and orientation (Sihvola, 1999). The Maxwell-Garnett mixing formula was chosen for use in this work because it allows the bulk properties of a medium to be determined simply by characterizing the inclusion with an array of dielectric spheres (Sihvola, 1999; Lindell et al., 1994; Long, 2001; Lakhtakia \& Weiglhofer, 1993).

Received 8 November 2004; accepted 31 May 2005.

The authors would like to thank Joshua S. Petko for his assistance with preparing the figures used in this manuscript.

Address correspondence to D. H. Werner, The Pennsylvania State University, Department of Electrical Engineering, University Park, PA 16802, USA. E-mail: dhw@psu.edu 

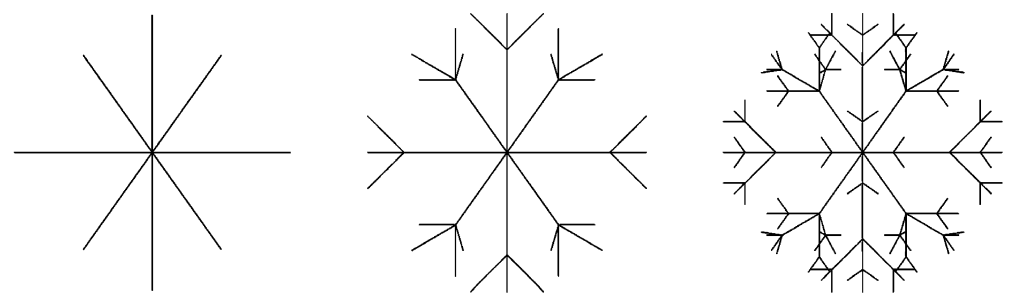

Figure 1. The first three iterations of a fractal sphere as viewed along the $x, y$, or $z$ axis.

This article investigates the electromagnetic backscattering properties of a new type of inclusion called a fractal sphere. A fractal sphere is a collection of symmetric selfsimilar fractal tree dipoles arranged to form a sphere-like structure as illustrated in Figure 1. It is demonstrated here that self-similar fractal inclusion geometries may be used to develop artificial dielectric metamaterials that are multi-band. It is also demonstrated that fractal spheres have a lower resonant frequency than solid spheres of the same physical size. Hence, another potentially important application of fractal spheres may be in the development of a class of artificial dielectric metamaterials for lowfrequency applications, since the lowest design frequency of such metamaterials is primarily dictated by the physical size of their molecules. This unique property of fractal sphere molecules may also lead to more compact (i.e., thinner) metamaterials for higher frequency applications.

\section{The Geometry of Fractal Spheres}

Many fractals of practical interest possess a geometrical structure that is self-similar (Peitgen, Jurgens, \& Saupe, 1992; Mandelbrot, 1983). This property of self-similarity has been recently exploited in the design of multi-band antennas (Werner \& Mittra, 2000; Gianvittorio \& Rahmat-Samii, 2002; Werner \& Ganguly, 2003) and multi-band frequency selective surfaces (Werner \& Ganguly, 2003; Romeu \& Rahmat-Samii, 2000; Werner \& Lee, 2000). In this article we investigate the backscattering of a new type of three-dimensional self-similar object called a fractal sphere. These fractal spheres are considered as potential candidates for molecules that can be used to synthesize multi-band artificial dielectric metamaterials.

Fractal spheres are composed of a series of nonintersecting self-similar fractal tree elements that extend outward from a common base point. Tree-like structures are one of the most common fractal shapes found in nature (Mandelbrot, 1983). For example, they have been observed in the geometry of river systems, undersea coral reefs, and vascular systems of the human body. The actual motivation for the fractal sphere introduced here resulted from a careful study of the bronchi of the lungs. It has been shown that the bronchi of the lungs are nearly self-similar over 15 successive iterations (Comroe, 1966). The fractal sphere is self-avoiding, meaning that as successive iterations are applied to the initiator base, the structure will not intersect itself at any point as it fills space.

The geometry of a three-dimensional fractal sphere is constructed by starting with a base initiator followed by successive iterations of an appropriately chosen generator. In the example considered here, the initiator consists of seven crossed dipoles, each assumed to be $5 \mathrm{~mm}$ in length. Three dipoles are oriented along the $x, y$, and $z$ axes, respectively, centered at the origin. The remaining four dipoles are arranged in an hourglass type of configuration, each displaced $45^{\circ}$ from the $z$ axis, with centers intersecting the origin. 
Next, a tree-like structure is formed by attaching four generator wires to each end of every dipole. These generator wires are positioned such that they make a $135^{\circ}$ angle with the base dipole and a $45^{\circ}$ angle with each of their neighbors. Each new application of these generators results in segment lengths that are half as long as they were for the previous iteration. The lengths of the first set of generator wires in this case were chosen to be $1.25 \mathrm{~mm}$. The first three iterations of this fractal sphere are shown in Figure 1. The first iteration shown consists of the initiator geometry only. The geometries for the second and third iterations include the first two applications of the generators, respectively, to the base geometry. Hence, as the number of iterations is increased, the wire segments will begin to effectively fill three-dimensional space without intersecting, thereby creating a self-avoiding fractal sphere.

\section{Scattering from a Fractal Sphere Molecule}

The backscatter cross-section for the first three iterations of the fractal sphere shown in Figure 1 was computed via a numerically rigorous wire scattering code based on the method of moments (MoM) (Harrington, 1968; Peterson, Ray, \& Mittra, 1998). A single fractal sphere molecule was placed in free space and excited by an incident plane wave. A $z$-polarized incident electric field propagating in the $-x$ direction will be considered in the analysis. The frequency range of interest spans from $1 \mathrm{GHz}$ to $200 \mathrm{GHz}$ in $1 \mathrm{GHz}$ intervals.

The plots of backscatter cross-section versus frequency are shown for the first through third iterations of the fractal sphere in Figures 2 through 4, respectively. Also shown on these plots for the sake of comparison is the backscatter cross-section resulting from a single linear dipole (Harrington, 1968) and a solid PEC sphere (Balanis, 1989). The single dipole is assumed to be $5 \mathrm{~mm}$ in length (i.e., the same size as the dipoles of the initiator), while the solid PEC sphere is assumed to have the same radius as the fractal sphere. The plots shown in Figure 2 indicated that, in the case of the first iteration fractal sphere,

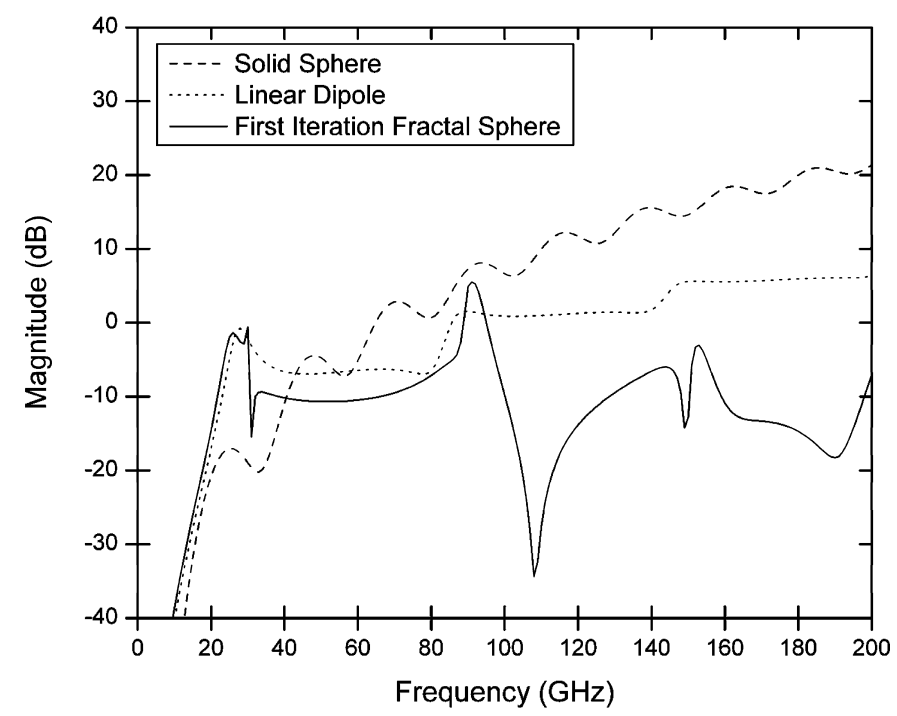

Figure 2. Backscatter cross-section versus frequency for first iteration fractal sphere compared to the response of a solid sphere of equivalent radius and a single linear dipole. 


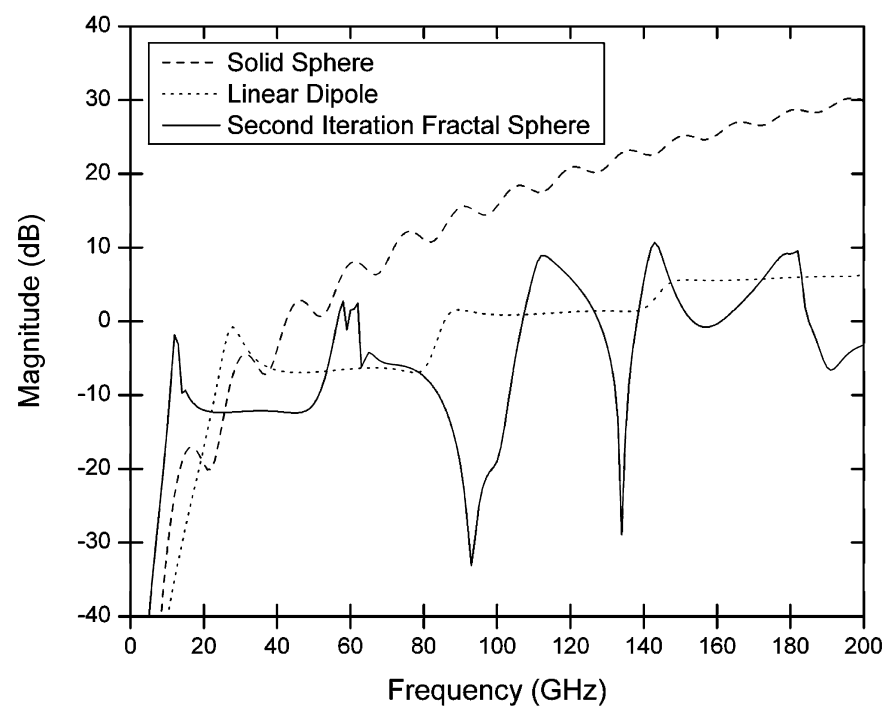

Figure 3. Backscatter cross-section versus frequency for second iteration fractal sphere compared to the response of a solid sphere of equivalent radius and a single linear dipole.

the first resonance of the solid sphere, single dipole, and first iteration fractal sphere structures coincide at $25 \mathrm{GHz}$. Similarities between the response of the first iteration fractal sphere and the single linear dipole are also evident from the plots shown in Figure 2. Next we consider Figure 3, which shows a plot of the backscatter cross-section versus frequency for the second iteration fractal sphere, where geometry is depicted in Figure 1. The plots included in Figure 3 demonstrate that the first resonance of the fractal sphere shifts to a lower frequency of $12.5 \mathrm{GHz}$, while the first resonance of

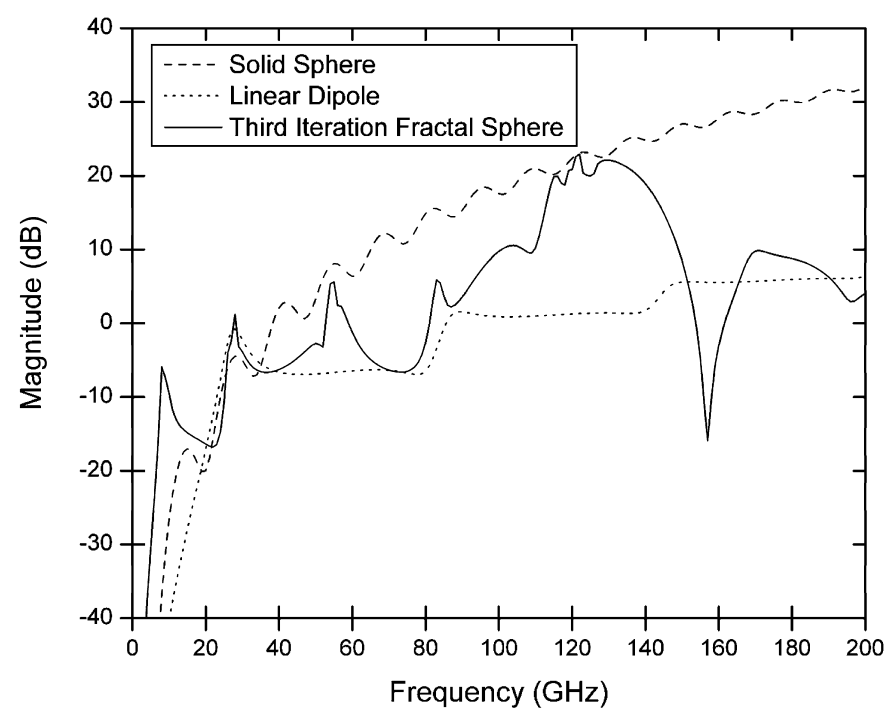

Figure 4. Backscatter cross-section versus frequency for third iteration fractal sphere compared to the response of a solid sphere of equivalent radius and a single linear dipole. 
Table 1

Size comparison of a solid sphere to an equivalent fractal sphere for the first three iterations

\begin{tabular}{cccr}
\hline Iteration & $\begin{array}{c}\text { Radius of fractal } \\
\text { sphere }(\mathrm{mm})\end{array}$ & $\begin{array}{c}\text { Radius of solid } \\
\text { sphere }(\mathrm{mm})\end{array}$ & $\begin{array}{c}\text { Percent size } \\
\text { increase }\end{array}$ \\
\hline 1 & 2.50 & 2.50 & 0.00 \\
2 & 3.49 & 5.00 & 43.27 \\
3 & 3.67 & 7.50 & 104.36 \\
\hline
\end{tabular}

the corresponding solid sphere occurs at a higher frequency of $17.5 \mathrm{GHz}$. Finally, the backscatter cross-section for the third iteration fractal sphere, the single $5 \mathrm{~mm}$ linear dipole, and the solid sphere of equivalent radius are plotted in Figure 4. In this case we find that the first resonance of the fractal sphere and the corresponding solid sphere shift down to $7.5 \mathrm{GHz}$ and to $15 \mathrm{GHz}$, respectively. Another very interesting feature of the third iteration fractal sphere is the region of enhanced backscatter between about 110 $\mathrm{GHz}$ and $130 \mathrm{GHz}$ shown in Figure 4. Within this frequency band the backscatter crosssection of the fractal sphere behaves in a way that is very similar to the solid sphere. Hence, as can be seen from Figure 4, the third iteration fractal sphere exhibits scattering properties of both the single linear dipole and the solid sphere.

A numerical comparison of the size reduction of the fractal sphere over the solid sphere is provided in Table 1 . The table is compiled by designing a solid sphere, regardless of radius, whose first resonance aligns with the first resonance of the fractal spheres as seen in Figures 2 through 4 (i.e., for the first, second, and third iterations). We see that the first resonances align in Figure 2 so that the radius of the solid sphere and fractal sphere are equivalent and there is no difference in size. Now, we consider a second-iteration fractal sphere with a radius of $3.49 \mathrm{~mm}$ (see Figure 3); it would require a solid sphere with a radius of $5 \mathrm{~mm}$ to properly align the first resonances. This suggests that, in this case, the fractal sphere would be $43 \%$ smaller in size than its conventional solid sphere counterpart. The effect is even more pronounced for the third iteration fractal sphere, with radius $3.67 \mathrm{~mm}$ (see Figure 4), where a solid sphere of radius $7.50 \mathrm{~mm}$ is required to align the resonances. This results in an over $104 \%$ size reduction of the third-iteration fractal sphere as compared to the more conventional solid sphere. This data shows that fractal spheres, although physically small, can appear to be electrically large.

\section{The Influence of Generator Scaling}

The next aspect of the fractal sphere that will be examined is the effect of the generator size on the scattering. All of the fractal spheres considered thus far were created from a half-scale generator. In other words, for each successive application of the generator, the length of the branches is reduced to half that of the previous base. We now examine what happens to the scattering properties of the fractal sphere if a quarter-scale instead of a half-scale generator is used. In this case the length of the branches for each new stage are reduced by a factor of 4 . The physical outcome is that the quarter-scale fractal fills the three-dimensional space less effectively than the half-scale fractal sphere. The progression for the first three iterations of the quarter-scale fractal sphere is shown in Figure 5. 

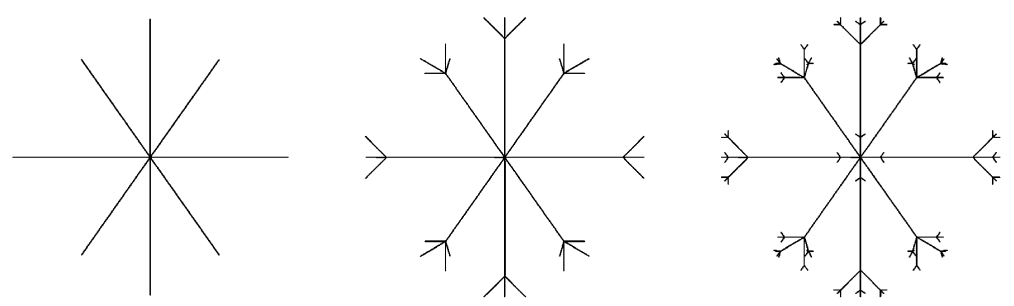

Figure 5. Evolution of a fractal sphere through three iterations based on a quarter-scale generator.

The effects of the generator modification are shown in Figure 6 as a comparison of backscatter cross-section versus frequency for the half-scale and quarter-scale third iteration fractal spheres. There are two interesting features to note in the backscatter curves of Figure 6. We can see that the first resonance of the quarter-scale fractal sphere occurs at a higher frequency than that of the half-scale fractal sphere. This higher resonant frequency is a consequence of the fact that the end-loading effects of the quarter-scale fractal sphere are not as pronounced as they are for the half-scale fractal sphere. The second interesting feature observed in the plots of Figure 6 is that the quarter-scale fractal sphere does not produce a region of enhanced backscatter like the half-scale fractal sphere does. This behavior can be attributed to the fact that the half-scale fractal sphere is more dense and therefore appears more like a solid sphere from an electromagnetics point of view. We may conclude from this analysis that the choice of the generator can greatly affect the scattering performance of fractal spheres.

\section{Ideal Dipole Approximation}

Suppose that the first fractal sphere is divided into a sequence of segments, each of which is assumed to be an ideal dipole. Hence, the total scattered field produced by an arbitrary

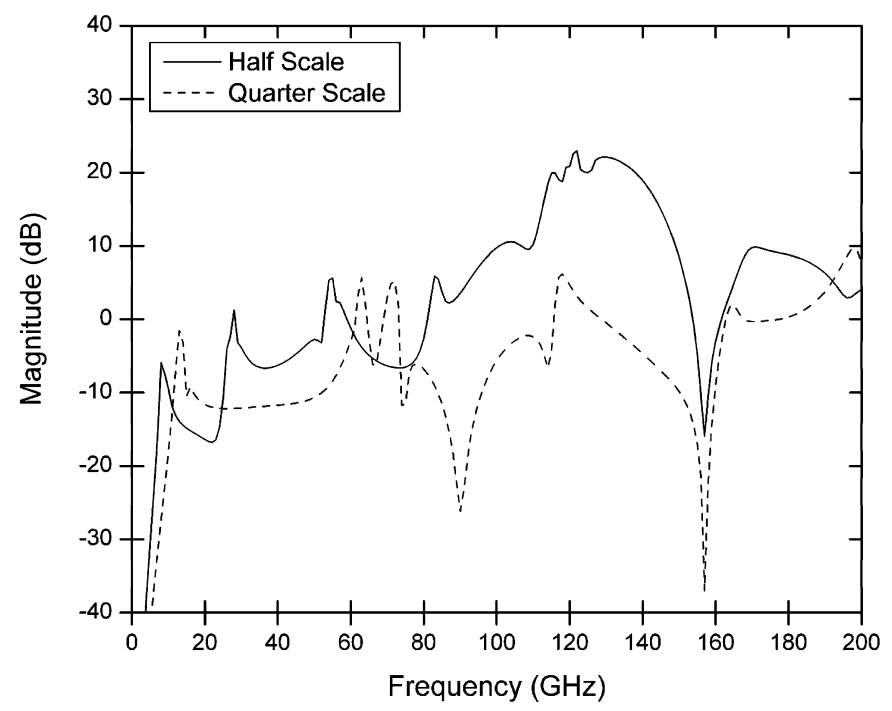

Figure 6. Backscatter cross-section versus frequency plot for third iteration quarter-scale and halfscale fractal spheres. 
segment of the fractal sphere centered at the point $\left(x_{o}^{\prime}, y_{o}^{\prime}, z_{o}^{\prime}\right)$ may be represented by (Jackson, 1966; Facchine, 2003)

$$
\mathbf{E}_{s c}^{T}(r, \theta, \varphi)=e^{-j k\left(x_{o}^{\prime} \sin \theta \cos \varphi+y_{o}^{\prime} \sin \theta \sin \varphi+z_{o}^{\prime} \cos \theta\right)} \mathbf{E}_{s c}(r, \theta, \varphi),
$$

where

$$
\mathbf{E}_{s c}(r, \theta, \varphi)=\frac{k^{2}}{4 \pi \varepsilon_{0}} \frac{e^{-j k r}}{r}[(\hat{\mathbf{r}} \times \mathbf{p}) \times \hat{\mathbf{r}}-(\hat{\mathbf{r}} \times \mathbf{m})] .
$$

The electric dipole moment $\mathbf{p}$ and the magnetic dipole moment $\mathbf{m}$, which appear in (2), may be written in the general form (Jackson, 1966)

$$
\begin{aligned}
& \mathbf{p}=\frac{1}{j \omega} \int_{S} \mathbf{J} d S^{\prime}, \\
& \mathbf{m}=\frac{1}{2 c} \int_{S}\left(\mathbf{r}^{\prime} \times \mathbf{J}\right) d S^{\prime},
\end{aligned}
$$

where $\mathbf{r}$ is defined as the vector from the origin to the observation point, $\mathbf{r}^{\prime}$ is defined as the vector from the origin to the source point, and $\hat{\mathbf{r}}$ is the unit normal vector in the direction of $\mathbf{r}$. In the case of an arbitrarily oriented ideal dipole segment of length $l$ and current distribution I, expressions (3) and (4) may be further simplified to (Facchine, 2003)

$$
\begin{aligned}
& \mathbf{p}=\frac{1}{j \omega} \int_{-\frac{l}{2}}^{\frac{l}{2}} \mathbf{I} d l^{\prime}, \\
& \mathbf{m}=\frac{1}{2 c} \int_{-\frac{l}{2}}^{\frac{l}{2}}\left(\mathbf{r}^{\prime} \times \mathbf{I}\right) d l^{\prime},
\end{aligned}
$$

where

$$
\begin{aligned}
\hat{\mathbf{r}} & =\sin \theta \cos \varphi \hat{\mathbf{x}}+\sin \theta \sin \varphi \hat{\mathbf{y}}+\cos \theta \hat{\mathbf{z}}, \\
\mathbf{r}^{\prime} & =x^{\prime} \hat{\mathbf{x}}+y^{\prime} \hat{\mathbf{y}}+z^{\prime} \hat{\mathbf{z}}, \\
x^{\prime} & =l^{\prime} \sin \alpha \cos \gamma, \\
y^{\prime} & =l^{\prime} \sin \alpha \sin \gamma, \\
z^{\prime} & =l^{\prime} \cos \alpha, \\
l^{\prime} & =\sqrt{\left(x^{\prime}\right)^{2}+\left(y^{\prime}\right)^{2}+\left(z^{\prime}\right)^{2}},
\end{aligned}
$$

and where $\alpha$ is the inclination angle of the ideal dipole measured from the $z$ axis and $\gamma$ is the rotation angle measured from the $x$ axis. Under these conditions, the expression given in (2) is a valid approximation provided the quantity

$$
k\left(\hat{\mathbf{r}} \bullet \mathbf{r}^{\prime}\right)=k l^{\prime}[\sin \theta \sin \alpha \cos (\varphi-\gamma)+\cos \theta \cos \alpha]
$$

is sufficiently small. 


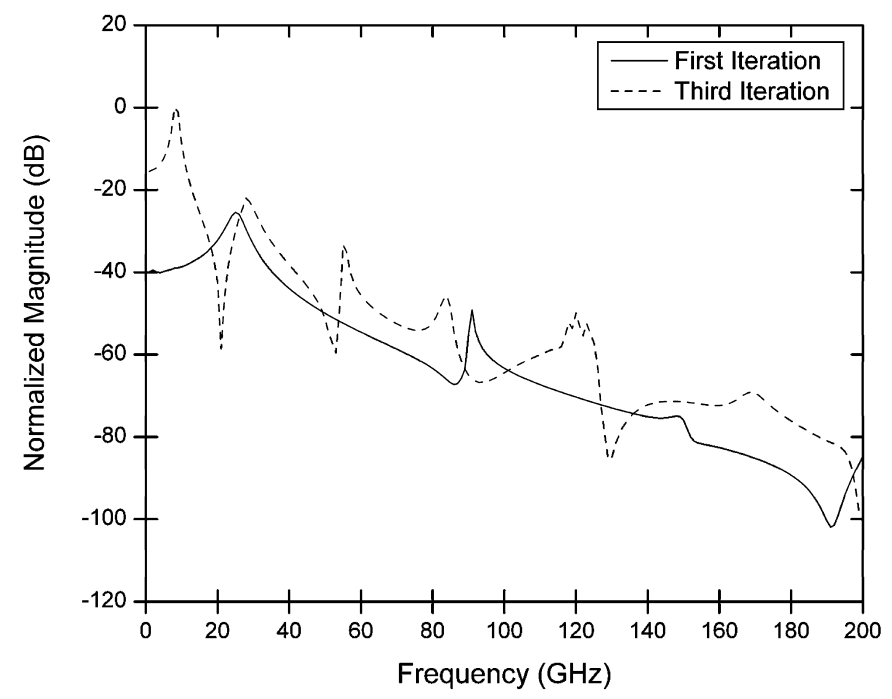

Figure 7. Normalized fractal sphere electric dipole moment for the first and third iterations. The maximum value of the electric dipole moment for the third iteration fractal sphere is $2.13 \mathrm{e}-11$ coul-m.

The electric and magnetic dipole moments can be used to calculate the effective media parameters of an artificial dielectric metamaterial with fractal spheres as inclusions in the host medium, or in fact, for any other type of desired inclusion. The values of the current distribution required in (5) and (6) are determined by first modeling the fractal sphere molecule using a rigorous full-wave MoM technique. Figure 7 shows plots of the normalized electric dipole moment for the third and first iterations of the fractal sphere. We can see the same resonance behavior in these plots as we have seen in the previous backscatter plots for the single fractal sphere molecule as computed via MoM simulations.

\section{Effective Dielectric Constant}

Metamaterials composed of fractal sphere molecules are too complicated for direct numerical evaluation. Instead, the scattering behavior of the individual molecules may be accurately represented by their electric and magnetic dipole moments. This allows the effective media parameters, permittivity and permeability, to be determined by considering a specific inclusion volume density for the host material and an appropriate mixing rule, such as the Maxwell-Garnett method (Sihvola, 1999).

Once the microscopic scattering properties of a particular molecule have been determined, the fractal sphere in this case, then it is possible to find the macroscopic properties of the artificial dielectric metamaterial hosting these inclusions. The derivation for the effective parameters begins from the knowledge that small particles of complex shape can be characterized by dyadic electric and magnetic polarizabilities, which relate the electric and magnetic dipole moments to the external electric and magnetic fields. These relationships are well known and further details concerning their derivation can be found elsewhere in (Sihvola, 1999; Mariotte, Sauviac, \& Tretyakov, 2000).

Solving for the effective media parameters requires the specification of an inclusion volume density along with knowledge of the polarizabilities of the inclusion. A somewhat conservative maximum inclusion packing density sets the inclusion spacing no smaller 
Table 2

Maximum number of inclusions per unit volume for each fractal sphere iteration

\begin{tabular}{lcc}
\hline Iteration & $\begin{array}{c}\text { "Shell" radius } \\
(\mathrm{mm})\end{array}$ & $\begin{array}{c}\text { Maximum number of } \\
\text { inclusions }\left(\mathrm{m}^{3}\right)\end{array}$ \\
\hline First & 2.50 & $7,944,997$ \\
Second & 3.49 & $2,920,364$ \\
Third & 3.67 & $2,511,350$ \\
\hline
\end{tabular}

than the maximum inclusion dimension. In solving for the maximum number of inclusions for the fractal spheres, we enclose the fractal sphere with a spherical "shell" that is of the same radius as the fractal sphere itself and proceed to use a simple sphere packing calculation. The sphere-packing algorithm utilized staggers the stacking axis in a way that is analogous to the classic supermarket pyramidal orange stack, allowing for maximum packing density to be achieved. The maximum number of inclusions per unit volume that was calculated using this sphere-packing algorithm is indicated in Table 2 for the first three iterations of the fractal sphere.

At this point we will examine the properties of an artificial dielectric metamaterial composed of fractal sphere molecules. For the purpose of this investigation we will assume the packing density of the metamaterial is determined by the maximum number of molecules that can be placed in a cubic meter volume without intersection (see Table 2). The resultant real and imaginary parts of the effective permittivity are shown in Figures 8 and 9, respectively, for a metamaterial comprising first iteration, second iteration, and third iteration fractal spheres. Also shown for comparison purposes are plots of the effective permittivity for an artificial dielectric metamaterial consisting of conventional linear dipole inclusions with a number density equivalent to the first iteration fractal sphere. These plots demonstrate that as the iteration of the fractal sphere molecules is increased, the corresponding lowest frequency band of the artificial dielectric metamaterial decreases. It is also observed that by using third iteration fractal sphere molecules, a dual-band artificial dielectric metamaterial can be created. Figures 10 and 11 show expanded views of Figures 8 and 9 for the frequency range 20-35 GHz. It is easily seen in Figures 10 and 11 that the second resonance of the dual-band metamaterial with third iteration fractal spheres is stronger than the first resonance of the single-band metamaterial made up of conventional linear dipoles.

\section{Conclusions}

The electromagnetic properties of fractal spheres and artificial dielectric metamaterials composed of fractal sphere molecules have been investigated. It was found that the self-similar geometrical structure of these fractal spheres produced a characteristic multiband response in their backscattering cross-section. This was a direct consequence of the fractal geometry with different self-similar parts of the structure resonating at different frequencies. It was also demonstrated that the fractal spheres possess backscattering properties that are characteristic of both linear dipoles and solid spheres. Moreover, it was shown that fractal spheres have a lower resonant frequency than solid spheres of the same physical size. The third iteration fractal sphere was physically half the size of a solid sphere with similar low-frequency resonance behavior. 


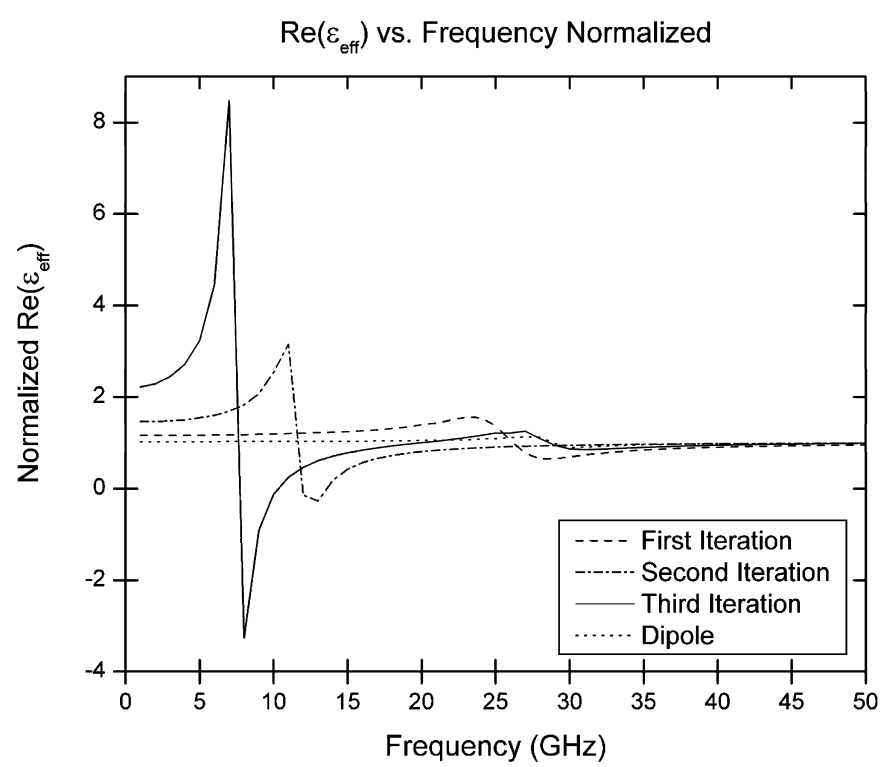

Figure 8. Normalized real part of the effective permittivity for an artificial dielectric metamaterial composed of fractal spheres with maximum number of inclusions.

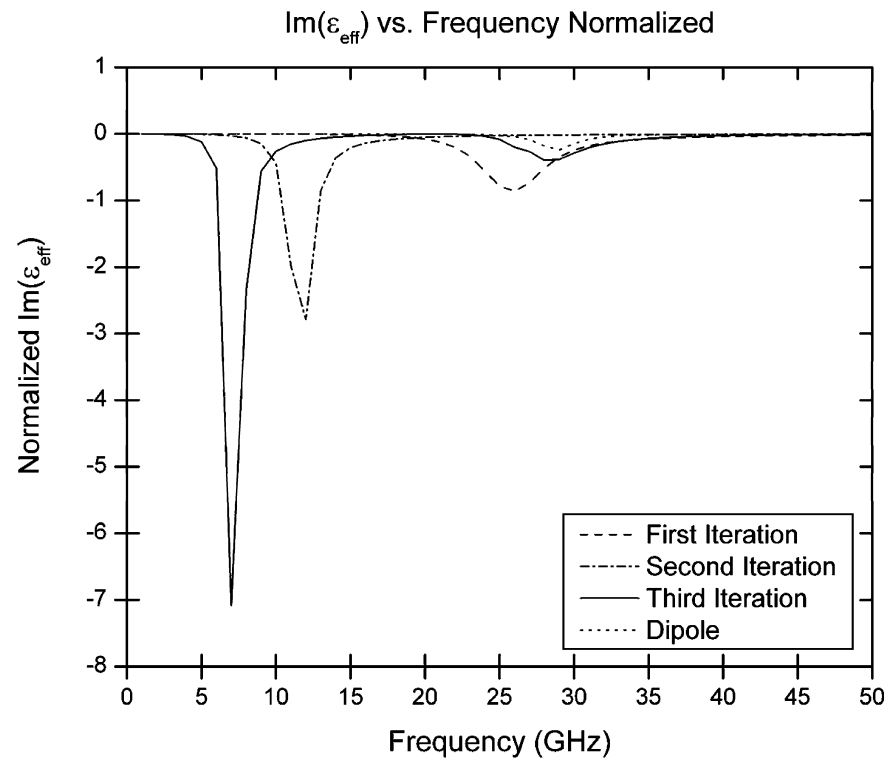

Figure 9. Normalized imaginary part of the effective permittivity for an artificial dielectric metamaterial composed of fractal spheres with maximum number of inclusions. 


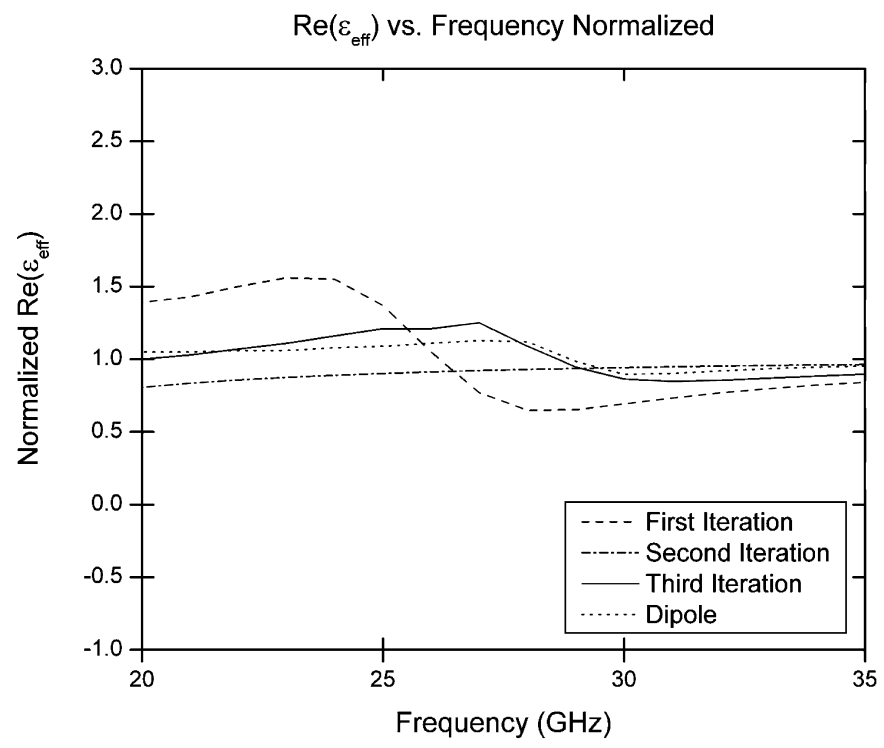

Figure 10. Normalized real part of permittivity for a $z$-oriented dipole compared to that of a first, second, and third iteration fractal sphere with maximum number of inclusions. Zoom on 20-35 $\mathrm{GHz}$ range.

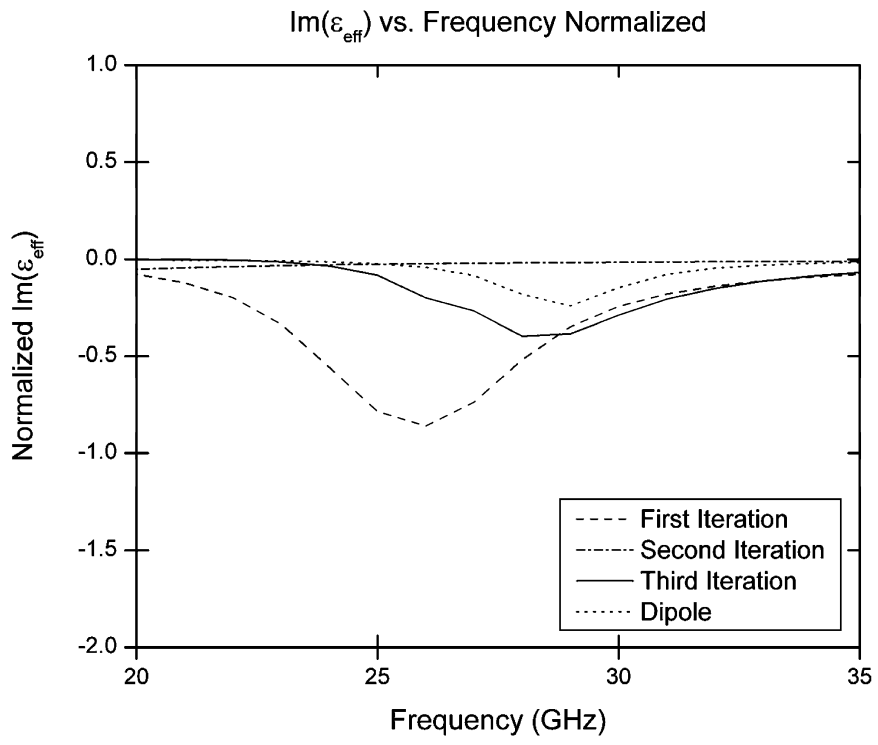

Figure 11. Normalized imaginary part of permittivity for a $z$-oriented dipole compared to that of a first, second, and third iteration fractal sphere with maximum number of inclusions. Zoom on 20-35 GHz range. 
Finally, the effective media parameters were extracted for an artificial dielectric metamaterial composed of fractal sphere molecules. A metamaterial composed of third iteration fractal spheres was shown to have a dual-band resonance behavior, the first resonance being much greater in magnitude and more pronounced than the second. The behavior of the effective media parameters reinforced the notion that these fractal spheres can be exploited as inclusions to develop a new class of low-frequency multi-band artificial dielectric metamaterials.

\section{References}

Bahr, A. J., \& K. R. Clausing. 1994. An approximate model for artificial chiral material. IEEE Transactions on Antennas and Propagation 42:1592-1598.

Balanis, C. A. 1989. Advanced engineering electromagnetics. New York: Wiley.

Comroe, J. H. 1966. The lung. Scientific American 214:56-68.

Facchine, M. 2003. The Electromagnetic properties of artificial dielectric metamaterials composed of fractal sphere molecules. MSEE Thesis, The Pennsylvania State University, University Park, PA.

Gianvittorio, J. P., \& Y. Rahmat-Samii. 2002. Fractal antennas: A novel antenna miniaturization technique, and applications. IEEE Antennas and Propagation Magazine 44:20-36.

Harrington, R. F. 1961. Time-harmonic electromagnetic fields. Toronto, Canada: McGraw-Hill.

Harrington, R. F. 1968. Field computation by moment methods. Malabar, FL: Robert E. Krieger.

Jackson, J. D. 1966. Classical electrodynamics. New York: Wiley.

Lakhtakia, A., \& W. S. Weiglhofer. 1993. Maxwell-Garnett estimates of the effective properties of a general class of discrete random composites. Acta Cryst. A49:266-269.

Lindell, I. V., A. H. Sihvola, S. A. Tretyakov, \& A. J. Viitanen. 1994. Electromagnetic waves in chiral and bi-isotropic media. Boston: Artech House.

Long, B. R. 2001. Analysis of stable negative impedance loaded dipole and canonical chiral elements with application to novel active media. Ph.D. Dissertation, Department of Electrical Engineering, The Pennsylvania State University, University Park, PA.

Mandelbrot, B. B. 1983. The fractal geometry of nature. New York: W. H. Freeman.

Mariotte, F., S. A. Tretyakov, \& B. Sauviac. 1996. Modeling effective properties of chiral composites. IEEE Antennas and Propagation Magazine 38:22-33.

Mariotte, F., B. Sauviac, \& S. A. Tretyakov. 2000. Artificial bianisotropic composites. In Frontiers in electromagnetics, ed. D. H. Werner and R. Mittra, pp. 732-770. Piscataway, NJ: IEEE Press.

Peitgen, H. O., H. Jurgens, \& D. Saupe. 1992. Chaos and fractals: New Frontiers of science. New York: Springer-Verlag.

Peterson, A., S. Ray, \& R. Mittra. 1998. Computational methods for electromagnetics. New York: IEEE Press.

Romeu, J., \& Y. Rahmat-Samii. 2000. Fractal FSS: A novel dual-band frequency selective surface. IEEE Transactions on Antennas and Propagation 48:1097-1105.

Sihvola, A. 1999. Electromagnetic mixing formulas and applications. London: The Institution of Electrical Engineers.

Werner, D. H., \& S. Ganguly. 2003. An overview of fractal antenna engineering research. IEEE Antennas and Propagation Magazine 45:38-57.

Werner, D. H., \& D. Lee. 2000. Design of dual-polarized multiband frequency selective surfaces using fractal elements. IEE Electronics Letters 36:487-488.

Werner, D. H., \& R. Mittra. 2000. Frontiers in electromagnetics. Piscataway, NJ: IEEE Press/Wiley. 